\title{
Conserved aspartic acids are essential for the enzymic activity of the WecA protein initiating the biosynthesis of O-specific lipopolysaccharide and enterobacterial common antigen in Escherichia coli
}

\author{
Amal O. Amer ${ }^{1}$ and Miguel A. Valvano ${ }^{1,2}$ \\ Author for correspondence: Miguel A. Valvano. Tel: +1 519661 3996. Fax: +1 5196613499. \\ e-mail:mvalvano@uwo.ca
}

Departments of

Microbiology and

Immunology ${ }^{1}$ and

Medicine2, University of

Western Ontario, London,

Ontario N6A 5C1, Canada

\begin{abstract}
The integral membrane protein WecA mediates the transfer of $\boldsymbol{N}$ acetylglucosamine (GICNAC) 1-phosphate to undecaprenyl phosphate (Und-P) with the formation of a phosphodiester bond. Bacteria employ this reaction during the biosynthesis of enterobacterial common antigen as well as of many O-specific lipopolysaccharides (LPSs). Alignment of a number of prokaryotic and eukaryotic WecA-homologous sequences identified a number of conserved aspartic acid (D) residues in putative cytoplasmic loops II and III of the innermembrane protein. Site-directed mutagenesis was used to study the role of the conserved residues D90, D91 (loop II), D156 and D159 (loop III). As controls, D35, D94 and D276 were also mutagenized. The resulting WecA derivatives were assessed for function by complementation analysis of $\mathbf{O}$-antigen biosynthesis, by the ability to incorporate radiolabelled precursor to a biosynthetic intermediate, by detection of the terminal GIcNAc residue in LPS and by a tunicamycin competition assay. It was concluded from these analyses that the conserved aspartic acid residues are functionally important, but also that they participate differently in the transfer reaction. Based on these results it is proposed that D90 and D91 are important in forwarding the reaction product to the next biosynthetic step, while D156 and D159 are a part of the catalytic site of the enzyme.
\end{abstract}

Keywords: undecaprenol, $\mathrm{N}$-acetylglucosamine, $\mathrm{O}$-antigen biosynthesis, membrane protein, phosphodiester bond

\section{INTRODUCTION}

Lipopolysaccharide (LPS) is a major component of the outer membrane in Gram-negative bacteria. It consists of lipid A, core oligosaccharide and the O-specific polysaccharide chain or $\mathrm{O}$ antigen (Whitfield, 1995). The biosynthesis of LPS is a complex process requiring many enzymes, encoded by a large number of genes, which catalyse the independent formation of a lipid A-core oligosaccharide complex and a polyisoprenyllinked $\mathrm{O}$ antigen. The biosynthesis and assembly of $\mathrm{O}$ antigen involves a characteristic transmembrane export of polyisoprenyl-linked saccharides that are synthesized

Abbreviations: GICNAC, $N$-acetylglucosamine; GPT, UDP-GICNAc: dolichol phosphate GICNAC-1-phosphate transferase; TMHMM, Transmembrane Hidden Markov Model; Und-P, undecaprenyl phosphate. on the cytoplasmic face of the plasma membrane. Depending on the components that may be required for this process, the assembly of $\mathrm{O}$ antigen can be distinguished into $w z y$-dependent and $w z y$-independent pathways (Whitfield, 1995). In $w z y$-dependent systems, the translocation of individual polyisoprenyl-linked $\mathrm{O}$ repeating oligosaccharide subunits to the periplasmic face of the plasma membrane is mediated by the Wzx protein (Feldman et al., 1999). Once on the periplasmic side, the individual subunits are polymerized by the Wzy O-antigen polymerase (Whitfield, 1995). In $w z y$-independent systems, polymerized $\mathrm{O}$ antigens are transported to the periplasmic face of the plasma membrane by an ATP-binding cassette transporter-dependent pathway (Bronner et al., 1994). Regardless of the specific export system used, polymerized $\mathrm{O}$ antigens are finally ligated onto the lipid A-core oligosaccharide acceptor 
and the complete LPS molecule is translocated to the outer membrane by an as yet undetermined mechanism (Gaspar et al., 2000).

The biochemical reactions to synthesize the $\mathrm{O}$ repeat occur in the cytoplasm/plasma membrane interface. They involve activated nucleotide sugar precursors that are available in the cytoplasm and membrane-associated glycosyltransferases that catalyse the formation of $\mathrm{O}$ glycosidic linkages. The synthesis of the $\mathrm{O}$ repeating subunit begins with the transfer of a sugar-1-phosphate residue to undecaprenyl phosphate (Und-P) forming an Und-P-P-linked saccharide intermediate. This intermediate serves as an acceptor for the glycosyltransferasedependent additions of the subsequent sugars to complete the synthesis of the $\mathrm{O}$ repeat. Various studies have shown that in many E. coli $\mathrm{O}$ antigens, the initiating enzyme is a tunicamycin-sensitive UDP-GlcNAc: Und-P GlcNAc-1-P transferase (Alexander \& Valvano, 1994; Klena \& Schnaitman, 1993; Rick \& Silver, 1996). This enzyme also initiates the synthesis of enterobacterial common antigen (Rick \& Silver, 1996) as well as O antigens in Shigella flexneri (Yao \& Valvano, 1994), Klebsiella pneumoniae (Clarke et al., 1995) and some of the Salmonella enterica serogroups (Rick \& Silver, 1996). Genetic and biochemical evidence supports that $w e c A$ (formerly $r f e$ ) is the structural gene encoding the UDP-GlcNAc: Und-P GlcNAc-1-P transferase (Rick \& Silver, 1996). The presence of alternating hydrophobic and hydrophilic domains in the amino acid sequence of E. coli WecA suggests that the protein spans the plasma membrane several times. In a previous study, we have shown that WecA is only present in a fraction containing plasma membrane markers, confirming that it is an integral membrane protein (Amer \& Valvano, 2000).

Significant sequence similarity occurs between E. coli WecA and other bacterial polyisoprenyl phosphate: $N$ acetylhexosamine-1-P transferases such as MraY, WbcO, RgpG and WbpL (Amer \& Valvano, 2001; Anderson et al., 2000; Dal Nogare \& Lehrman, 1988; Lehrman, 1994). This protein family also includes the eukaryotic UDP-GlcNAc: dolichol phosphate GlcNAc1-P transferases (GPTs), which catalyse the first step in the biosynthesis of the glycan moiety of glycoproteins (Burda \& Aebi, 1999). The hamster GPT has been characterized (Dan \& Lehrman, 1997; Dan et al., 1996; Datta \& Lehrman, 1993; Zhu \& Lehrman, 1990). A set of conserved sequences found in eukaryotic GPTs and in prokaryotic enzymes suggested a functional conservation of this enzyme family (Dal Nogare \& Lehrman, 1988), although eukaryotic GPTs function with dolichol-P as an acceptor instead of Und-P (Rush et al., 1997). Limited information is available on structural motifs and specific amino acids of WecA that may be important for substrate recognition and/or catalysis (Anderson et al., 2000). We have recently identified conserved amino acid residues in a predicted large cytosolic region of WecA, which may be implicated in the recognition of UDP-GlcNAc (Amer \& Valvano, 2001). In this study, we report the identification of two other regions of the E. coli WecA protein containing aspartic acids that are highly conserved within prokaryotic and eukaryotic members of this family and which are also part of predicted cytosolic loops. We provide evidence suggesting that the conserved aspartic acid residues may be required for the catalysis of the phosphodiester bond between Und-P and GlcNAc 1phosphate, probably via ionic interactions with divalent metal cations.

\section{METHODS}

Strains and plasmids. Table 1 summarizes the properties of $E$. coli strains and plasmids used in this study. In vivo complementation experiments were conducted with E. colis strain MV501, a wecA::Tn10 mutant of the E. coli O7 VW187 (Alexander \& Valvano, 1994). E. coli CLM20 was used to detect the ligation of a single GlcNAc residue onto the lipid A core as described elsewhere (Feldman et al., 1999). Bacteria were cultured in Luria-Bertani (LB) medium supplemented with $100 \mu \mathrm{g}$ ampicillin $\mathrm{ml}^{-1}, 20 \mu \mathrm{g}$ tetracycline $\mathrm{ml}^{-1}$ and $0.02 \%(\mathrm{w} / \mathrm{v}$ ) arabinose as appropriate. Chemical reagents were purchased from Sigma unless otherwise indicated.

Construction of pAA1 and pAA3. PCR was carried out with PwoI DNA polymerase (Roche Diagnostics) in a Hybaid Ommigene thermocycler (Interscience). The oligonucleotides used in this study are listed in Table 2. pAA1 was constructed by PCR amplification of a $1.4 \mathrm{~kb}$ fragment encompassing the wecA gene in pRL100. This fragment was amplified with the $5^{\prime}$ primer 62, incorporating an EcoRI site, and primer 63, encoding the 3 -terminal end of wecA. The amplified DNA product was digested with EcoRI and ligated to the EcoRI and SmaI sites of pGEM3. pAA3 was constructed following a similar strategy with primer 102 , encoding the 3 '-terminal part of $w e c A$ up to T1344 and an added XbaI site. The fragment was digested with EcoRI and XbaI, ligated into pGEM3 that was also digested with the same enzymes and the mixture was transformed into E. coli $\mathrm{DH} 5 \alpha$.

Oligonucleotide-directed mutagenesis of wecA. Plasmid pAA26, containing the wecA gene tagged with the FLAG sequence (Amer \& Valvano, 2000), was used as a template in all PCR reactions except for those involved in the construction of the DD156/159GG mutant, where the template was pAA45. pAA34 was constructed by PCR amplification of the $3^{\prime}$ end of $w e c A$ using primer 165 , which anneals to the FLAG sequence, and primer 254, which encodes a glycine residue substituting D277. The amplicon obtained was ligated into the SmaI site of pBAD24, followed by digestion with EcoRI and SmaI, and subsequent ligation with a fragment encoding the remaining $5^{\prime}$ end of the wecA gene that was amplified using primers 255 and 170. The other aspartic acid substitutions in the wecA gene were introduced directly by site-directed mutagenesis using the QuikChange Site-Directed Mutagenesis Kit from Stratagene, as recommended by the supplier. DNA fragments were purified from $0.7 \%$ agarose gels with the QIAquick Gel Extraction Kit (Qiagen) and ligated with T4 DNA ligase (Roche Diagnostics) to the corresponding plasmid vectors. Ligation products were transformed into E. coli $\mathrm{DH} 5 \alpha$ or XL-1 Blue, as appropriate, by the calcium chloride method (Hanahan, 1983). Replacement mutants were confirmed by sequencing the entire $w e c A$ gene with an automated sequencer at the DNA Sequencing Facility, Robarts Research Institute, London, Ontario, Canada.

In vitro transferase and binding assays. pAA26, encoding the $w e c A_{\mathrm{FLAG}}$ gene, and the plasmids encoding the mutated wecA 
Table 1. Bacterial strains and plasmids used in this study

\begin{tabular}{|c|c|c|}
\hline $\begin{array}{l}\text { Strain or } \\
\text { plasmid }\end{array}$ & Relevant properties & Source or reference \\
\hline \multicolumn{3}{|l|}{ Strain } \\
\hline CLM20 & lacZ $\operatorname{trp} \Delta(s b c B-r f b)$ upp rel $r p s L$ recA & Feldman et al. (1999) \\
\hline DH5 $\alpha$ & end $A$ hsdR supE thi-1 recA relA gyrA & Laboratory stock \\
\hline MV501 & VW187; wecA::Tn10 Tet $^{\mathrm{R}}$ & Alexander \& Valvano (1994) \\
\hline XL-1 Blue & $\begin{array}{l}\text { recA end } \text { gyrA thi-1 hsdR supE relA lac }\left[\mathrm{F}^{\prime}\right. \\
\left.\text { pro } A B \text { lacl }^{\mathrm{q}} \mathrm{Z} \Delta \mathrm{M} 15 \mathrm{Tn} 10\left(\mathrm{Tet}^{\mathrm{R}}\right)\right]\end{array}$ & Stratagene \\
\hline VW187 & $\mathrm{O} 7$; $\mathrm{K} 1$; clinical isolate & Valvano \& Crosa (1989) \\
\hline \multicolumn{3}{|l|}{ Plasmid } \\
\hline pAA1 & $\begin{array}{l}1.4 \mathrm{~kb} \text { fragment containing } w e c A \text { cloned in } \\
\text { pGEM } 3 ; \mathrm{Amp}^{\mathrm{R}}\end{array}$ & This study \\
\hline pAA3 & $1.3 \mathrm{~kb}$ fragment containing part of $w e c A ; \mathrm{Amp}^{\mathrm{R}}$ & This study \\
\hline pAA26 & $\begin{array}{l}1.2 \mathrm{~kb} \text { EcoRI-PvuII fragment from pAA14 } \\
\text { (Amer \& Valvano, 2000) encoding } w e c A_{\mathrm{FLAG}} \\
\text { ligated into EcoRI and SmaI sites of pBAD24; } \\
\text { Amp }^{\mathrm{R}}\end{array}$ & This study \\
\hline pAA34 & pAA26 encoding $w e c A_{\mathrm{D} 276 \mathrm{G}}$ & This study \\
\hline pAA35 & pAA26 encoding $w e c A_{\mathrm{D} 35 \mathrm{G}}$ & This study \\
\hline pAA36 & pAA26 encoding $w e c A_{\mathrm{DD} 90 / 91 \mathrm{GG}}$ & This study \\
\hline pAA37 & pAA26 encoding $w e c A_{\mathrm{DD} 90 / 91 \mathrm{EE}}$ & This study \\
\hline pAA40 & pAA26 encoding $w e c A_{\mathrm{DD} 156 / 159 \mathrm{GG}}$ & This study \\
\hline pAA41 & pAA26 encoding $w e c A_{\mathrm{DD} 156 / 191 \mathrm{EE}}$ & This study \\
\hline pAA43 & pAA26 encoding $w e c A_{\mathrm{D} 156 \mathrm{G}}$ & This study \\
\hline pAA45 & pAA26 encoding $w e c A_{\mathrm{D} 159 \mathrm{G}}$ & This study \\
\hline pAA47 & pAA26 encoding $w e c A_{\mathrm{D} 94 \mathrm{G}}$ & This study \\
\hline pBAD24 & Cloning vector, $\mathrm{Amp}^{\mathrm{R}}$ & Guzman et al. (1995) \\
\hline pGEM3 & Cloning vector, $\mathrm{Amp}^{\mathrm{R}}$ & Promega \\
\hline pMF21 & $\begin{array}{l}1.5 \mathrm{~kb} \text { fragment encoding } w z x_{\mathrm{EcO} 7} \text { cloned into } \\
\text { pEXT21, } \mathrm{Sp}^{\mathrm{R}}\end{array}$ & Feldman et al. (1999) \\
\hline pRL100 & $\begin{array}{l}1.46 \mathrm{~kb} \text { ClaI-XmaIII fragment containing wecA } \\
\text { cloned in pBR322; } \mathrm{Amp}^{\mathrm{R}}\end{array}$ & Meier-Dieter et al. (1992) \\
\hline
\end{tabular}

derivatives were transformed by electroporation (Dower et al., 1988) into E. coli strain MV501, which carries a wecA::Tn10 insertion (Alexander \& Valvano, 1994). Membranes (containing enzymes and endogenous Und-P) were isolated from these transformants as described by Osborn et al. (1972), following induction with $0.02 \%$ arabinose for $3 \mathrm{~h}$. The reaction mixture for the transferase assay contained the membrane fraction ( $20 \mu \mathrm{g}$ total protein), $96 \mathrm{pmol}$ radiolabelled UDP-N-acetyl- $\left[{ }^{14} \mathrm{C}\right]$ glucosamine (Amersham Pharmacia Biotech) in $250 \mu \mathrm{l}$ buffer ( $5 \mathrm{mM}$ Tris-acetate, $\mathrm{pH} 8.5,0.1 \mathrm{mM}$ EDTA, $1 \mathrm{mM} \mathrm{MgCl}$ ). After $30 \mathrm{~min}$ incubation at $37^{\circ} \mathrm{C}$, the lipid-associated material was extracted twice with $250 \mu \mathrm{l} 1$ butanol. The combined 1-butanol extracts were washed once with $500 \mu \mathrm{l}$ distilled water and the radioactive counts of the 1 butanol fraction were determined with a Beckman liquid scintillation counter. Radioactive counts were normalized by determining the amount of parental or mutated WecA proteins in the same membrane fractions used for the enzymic assay. Detection and quantification of WecA proteins was carried out by immunoblot analysis with anti-FLAG antibodies (see below), following densitometry. We also ensured that equal amounts of protein were loaded in the gels and small differences in loading were further corrected by reprobing the blots with anti-OmpA antibodies (see below). Enzyme activity was finally expressed as pmol incorporated GlcNAc (mg membrane protein $)^{-1}$. Under the conditions used, the enzyme activity of membrane extracts containing the parental $\mathrm{WecA}_{\mathrm{FLAG}}$ was linear between 10 and $40 \mu \mathrm{g}$ membrane protein. For comparisons between parental and mutated forms of WecA, enzyme activity was also expressed as the percentage of WecA $_{\mathrm{FLAG}}$ activity at $1 \mathrm{mM} \mathrm{MgCl}_{2}$ concentration.

Binding assays were performed as described by Dal Nogare $\&$ Lehrman (1988). This assay is based on the ability of the UDPGlcNAc analogue tunicamycin to bind and inhibit the enzymic activity of the parental WecA (Alexander \& Valvano, 1994; Barr et al., 1989) following incubation with membranes containing the WecA mutants. Thus, the level of transferase activity in this assay is a function of the residual concentration of tunicamycin. Forty micrograms of membranes from MV501 cells transformed with plasmids encoding the various wecA mutants was mixed with transferase buffer containing $0 \cdot 225 \mu \mathrm{g}$ tunicamycin $\mathrm{ml}^{-1}$ in a total volume of $200 \mu \mathrm{l}$ and incubated for $10 \mathrm{~min}$ at room temperature. The presence of residual tunicamycin failing to bind to the mutant proteins was determined by the standard transfer assay after the addition of $20 \mu \mathrm{g}$ MV501(pAA26) membranes and radiolabelled UDP-Nacetyl- $\left[{ }^{14} \mathrm{C}\right]$ glucosamine. We determined that under our experimental conditions $0 \cdot 225 \mu \mathrm{g}$ tunicamycin $\mathrm{ml}^{-1}$ are sufficient to inhibit the $\mathrm{WecA}_{\mathrm{FLAG}}$ parental activity of the membranes from MV501(pAA26). Enzyme activity was expressed as a 
Table 2. Oligonucleotide primers used in this study

\begin{tabular}{|c|c|c|}
\hline WecA mutant & Primer no. & Primer sequence $\left(5^{\prime}-3^{\prime}\right)^{*}$ \\
\hline & 62 & TCGATGCAATGGAAT \\
\hline & 63 & GGTTAAATTGGGGCTGC \\
\hline & 102 & CGCTCTAGACGCTTAATGCAATATCCAT \\
\hline & 165 & CTCGAGTCACTTGTCGTC \\
\hline & 170 & ATTACGAATTCGGTGAATTTACTGACAGTG \\
\hline \multirow[t]{2}{*}{$\mathrm{D} 276 \mathrm{G}$} & 254 & GGGCGTCAGCATATTCAC \\
\hline & 255 & 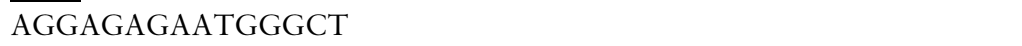 \\
\hline \multirow[t]{2}{*}{ DD90/91GG } & 289 & GCTTGTTTTCATTGGCGCGCTGGGTGGCCGTTTTGATATCAGC \\
\hline & 290 & GCTGATATCAAAACGGCCACCCAGCGCGCCAATGAAAACAAGC \\
\hline \multirow[t]{2}{*}{ DD90/91EE } & 420 & GCTTGTTTTCATTGGCGCGCTGGAAGAGCGTTTTGATATCAGC \\
\hline & 421 & GCTGATATCAAAACGCTCTTCCAGCGCGCCAATGAAAACAAGC \\
\hline \multirow[t]{2}{*}{ D94G } & 312 & GGCGCGCTGGATGACCGTTTTGGTATCAGCGTAAAAATCCGTGCC \\
\hline & 313 & GGCACGGATTTTTACGCTGATACCAAAACGGTCATCCAGCGCGCC \\
\hline \multirow[t]{2}{*}{ DD156/159GG } & 304 & GCGTTCAACATGGTTGGTGGCATTGGTGGCTGGC \\
\hline & 305 & 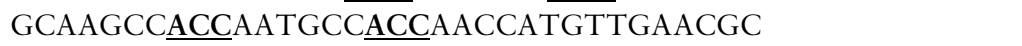 \\
\hline \multirow[t]{2}{*}{ D156G } & 314 & GCGTTCA ACATGGTTGGTGGCATTGATGGCTTGC \\
\hline & 315 & GCAAGCCATCAATGCCACCAACCATGTTGAACGC \\
\hline \multirow[t]{2}{*}{ D159G } & 291 & GCGTTCAACATGGTTGATGGCATTGGTGGCTTGCTGGGCGGG \\
\hline & 292 & CCCGCCCAGCAAGCCACCAATGCCATCAACCATGTTGAACGC \\
\hline \multirow[t]{2}{*}{ DD156/159EE } & 387 & 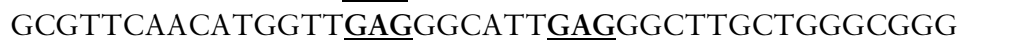 \\
\hline & 388 & 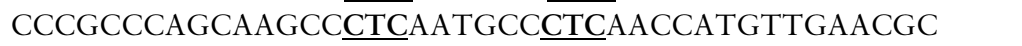 \\
\hline
\end{tabular}

* Sequences encoding the mutated codons are indicated in bold and underlined.

percentage of parental WecA activity assayed under the same conditions in the absence of tunicamycin.

Immunoblot analysis. Aliquots of membrane preparations were also used for immunoblot analysis. Two micrograms of membranes were mixed with the appropriate amount of loading sample buffer $(50 \mathrm{mM}$ Tris $/ \mathrm{HCl}, \mathrm{pH} 6 \cdot 8,2 \%$ SDS, $10 \%$ glycerol and $0 \cdot 1 \%$ bromophenol blue) and incubated at $45^{\circ} \mathrm{C}$ for $30 \mathrm{~min}$ before loading onto a $10 \%$ SDS-polyacrylamide gel. We demonstrated in a previous report that these conditions are optimal to detect WecA, since complete denaturation by boiling prevents its detection by immunoblotting (Amer \& Valvano, 2000). The transfer of protein to nitrocellulose membranes was performed according to standard procedures. Membranes were blocked overnight with $5 \%$ skim milk dissolved in TBS $(50 \mathrm{mM}$ Tris, $150 \mathrm{mM}$ sodium chloride, $\mathrm{pH} 7 \cdot 6$ ), incubated for $2 \mathrm{~h}$ with the FLAG M2 mAb at a concentration of $7 \mu \mathrm{g} \mathrm{ml}^{-1}$ and washed several times with TBS. This was followed by a $2 \mathrm{~h}$ incubation period with horseradish-peroxidase-linked sheep anti-mouse IgG (Amersham Pharmacia Biotechnology) at a dilution of $1: 3000$. Detection by chemiluminescence was performed using the BM Chemiluminescence Blotting Substrate (Roche Diagnostics), as recommended by the manufacturer. Loading was normalized by determining the relative amounts of OmpA in the membrane preparations using OmpA-specific rabbit antibodies. Nitrocellulose membranes previously reacted with FLAG $\mathrm{M} 2$ were incubated at $50{ }^{\circ} \mathrm{C}$ for $15 \mathrm{~min}$ in stripping buffer (Tris/ $\mathrm{HCl}, \mathrm{pH} 6 \cdot 8,2 \%$ SDS, 2\% 2-mercaptoethanol). This was followed by several washes with TBS, and overnight blocking and development as described above, except that the anti-OmpA primary antibody was used at a dilution of $1: 10000$ and the horseradish-peroxidase-linked sheep antirabbit IgG secondary antibody (Amersham Pharmacia Biotechnology) was at a dilution of $1: 3000$.
LPS analysis. LPS was extracted and analysed by SDS-PAGE followed by silver staining as described by Marolda et al. (1990). For determination of the incorporation of a terminal GlcNAc into the lipid A-core oligosaccharide, LPS from E. coli CLM20 containing pMF21 (encoding the O antigen translocase $\mathrm{Wzx}$ ) and the various plasmids encoding parental and mutated WecA constructs was analysed by immunoblotting using digoxigenin-labelled wheat germ agglutinin as described by Feldman et al. (1999).

Fractionation of membranes. Membranes were prepared and analysed by sucrose gradient fractionations as described previously (Amer \& Valvano, 2000, 2001)

Amino acid sequence alignments. BLAST version 2 (Altschul et al., 1997) was used to search the database of non-redundant sequences with WecA as a query. Amino acid sequence alignments of WecA homologues were performed using CLUSTAL w (Thompson et al., 1994). Transmembrane helices were predicted using the Dense Sequence Alignment method (Cserzo et al., 1997) and the Transmembrane Hidden Markov Model (TMHMM) method (Sonnhammer et al., 1998).

\section{RESULTS}

\section{Highly conserved aspartic acid residues in two regions of the WecA protein}

We have shown in a previous study that the predicted topology of WecA (Fig. 1a) is in good agreement with the topological model experimentally confirmed for $E$. coli MraY (Amer \& Valvano, 2001), which belongs to the WecA protein family. The agreement between the two topological models is especially strong within nontransmembrane segments with high amino acid sequence 
conservation (Fig. 1a, loops II, III and V), which are orientated towards the cytosolic compartment (Amer \& Valvano, 2001). We have also identified a region near the $\mathrm{C}$ terminus of the WecA protein (Fig. 1a, loop V) that may function in the recognition of UDP-GlcNAc (Amer \& Valvano, 2001). A comparison of multiple alignments of protein sequences from prokaryotic and eukaryotic WecA homologues revealed two additional regions containing several conserved aspartic acids (Fig. 1). One of these regions, represented by the peptide sequence I86-G87-A88-L89-D90-D91-R92-F92-D94 of the E. coli WecA protein, contains two highly conserved aspartic acids at positions 90 and 91 (Fig. 1b). From these, D91 was conserved in all WecA homologues, while D90 was present in all but three members of the family. The other conserved region contains the peptide sequence N150A151-F152-N153-M154-V155-D156-G157-I158-D159G160-L161 (Fig. 1). This peptide has an aspartic acid at position 156 that is conserved in the prokaryotic eubacterial homologues of WecA, while an alanine is found at the same position in the eukaryotic enzymes and the two archaeal homologues from Sulfolobus. D159 was conserved in the majority of eubacterial WecA homologues, while an asparagine was found in the remaining sequences (Fig. 1). The conserved aspartic acids of WecA, indicated in Fig. 1(b), are most likely part of cytosolic hydrophilic domains since they correspond exactly to residues at similar positions in the $E$. coli MraY protein (loops II and III). Both regions were shown to be exposed on the cytosolic face of the plasma membrane (Fig. 1; Bouhss et al., 1999). No glutamic acid residues were located in any of the regions of WecA that were predicted to be facing the cytosol.

To facilitate the functional analysis of WecA, we constructed a C-terminally FLAG-tagged WecA protein that could be followed by immunoblot analysis (Amer \& Valvano, 2000). Complementation experiments in $E$. coli MV501, which carries a wecA:: Tn10 insertion that prevents the formation of $\mathrm{O} 7$ polysaccharide (Alexander \& Valvano, 1994), indicated that the addition of the epitope tag plus a spacer region of 11 aa did not compromise the function of the recombinant protein (Amer \& Valvano, 2000). We also constructed a deletion of the wecA gene that eliminates the last 27 aa of WecA. The resulting recombinant plasmid, pAA3, was transformed into MV501. LPS from the transformants was prepared and examined by SDS-PAGE and silver staining. Fig. 2 (lane 4) shows that the deleted WecA can efficiently complement O7 LPS synthesis in MV501 at levels comparable to those of wild-type WecA (pAA1) as well as the parental E. coli O7 strain VW187. We concluded from this experiment that the C-terminal region of $\mathrm{WecA}$ is dispensable for function.

\section{Substitutions of conserved aspartic acid residues do not compromise expression and localization of WeCA $_{\text {FLAG }}$ in the plasma membrane}

Given that $\mathrm{WecA}_{\mathrm{FLAG}}$ is functionally comparable to the wild-type protein, the $w e c A_{\mathrm{FLAG}}$ gene was used as the parent for the construction and functional charac- terization of aspartic acid replacement mutants. The conserved aspartic acids at positions 90, 91, 156 and 159 were replaced with either glycine or glutamic acid by site-directed mutagenesis. Replacements of nonconserved aspartic acids at positions 35, 94 and 276, which are also predicted to correspond to cytosolic domains of the protein (Fig. 1a), were performed as a control. Glycine is a neutral non-hydrophobic residue, while glutamic acid, at physiological $\mathrm{pH}$, has a similar negative charge to aspartic acid. The possibility that the amino acid replacements could affect either protein stability or targeting to the plasma membrane was ruled out by examining the levels of expression of the various constructs in membrane preparations. Fig. 3 shows an immunoblot demonstrating that the majority of mutant proteins exhibit a level of expression similar to that of the parental WecA $\mathrm{FLAG}_{\mathrm{G}}$ encoded by pAA26. The higher bands observed in the immunoblots correspond to WecA oligomers, which are not completely denatured under the conditions used to detect the protein (Amer \& Valvano, 2000). As demonstrated in a previous report, mild denaturation is required for the visualization of WecA and the oligomers are likely the result of aggregation of WecA monomers, since the mass of the aggregates decreases proportionately in $\mathrm{N}$-terminal deleted variants of $\mathrm{WecA}_{\mathrm{FLAG}}$ (Amer \& Valvano, 2000). The localization of the mutant proteins in the plasma membrane was confirmed by the analysis of membrane fractions obtained following sucrose gradient centrifugation. All WecA mutants were localized in the fractions containing cytoplasmic membrane components, as defined by the presence of the plasma membrane marker NADH oxidase and lack of outermembrane porins (data not shown). The parental WecA $_{\text {FLAG }}$ and its mutated derivatives were not detected in the pellet of the sucrose gradients, ruling out the presence of inclusion bodies. Taken together, these results suggest that the aspartic acid replacements did not affect either expression or localization of the WecA ${ }_{\text {FLAG }}$ mutants.

\section{Effect of aspartic acid substitutions on the function of WecA}

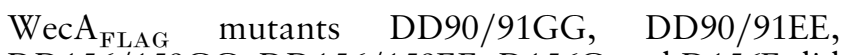
DD156/159GG, DD156/159EE, D156G and D156E did not complement the synthesis of O7 LPS in vivo (Fig. 4, lanes 4, 5, 7, 8 and 9, and data not shown). The in vitro transfer activity of membranes containing these proteins was dramatically reduced compared to the activity of membranes with the parental $\mathrm{WecA}_{\mathrm{FLAG}}$ (Table 3). D156G and DD156/159GG mutants gave similar levels of very low activity, while the D159G mutant showed a partial complementation of the O7 LPS-deficient phenotype (Fig. 4, lane 10), as well as a moderate level of transferase activity (Table 3). We concluded from these results that the low level of activity of the DD156/159GG mutant protein was caused by the replacement of D156, which possibly had a more drastic effect in the catalytic activity of WecA. The mutants DD90/91GG and DD90/91EE displayed higher levels 
(a)
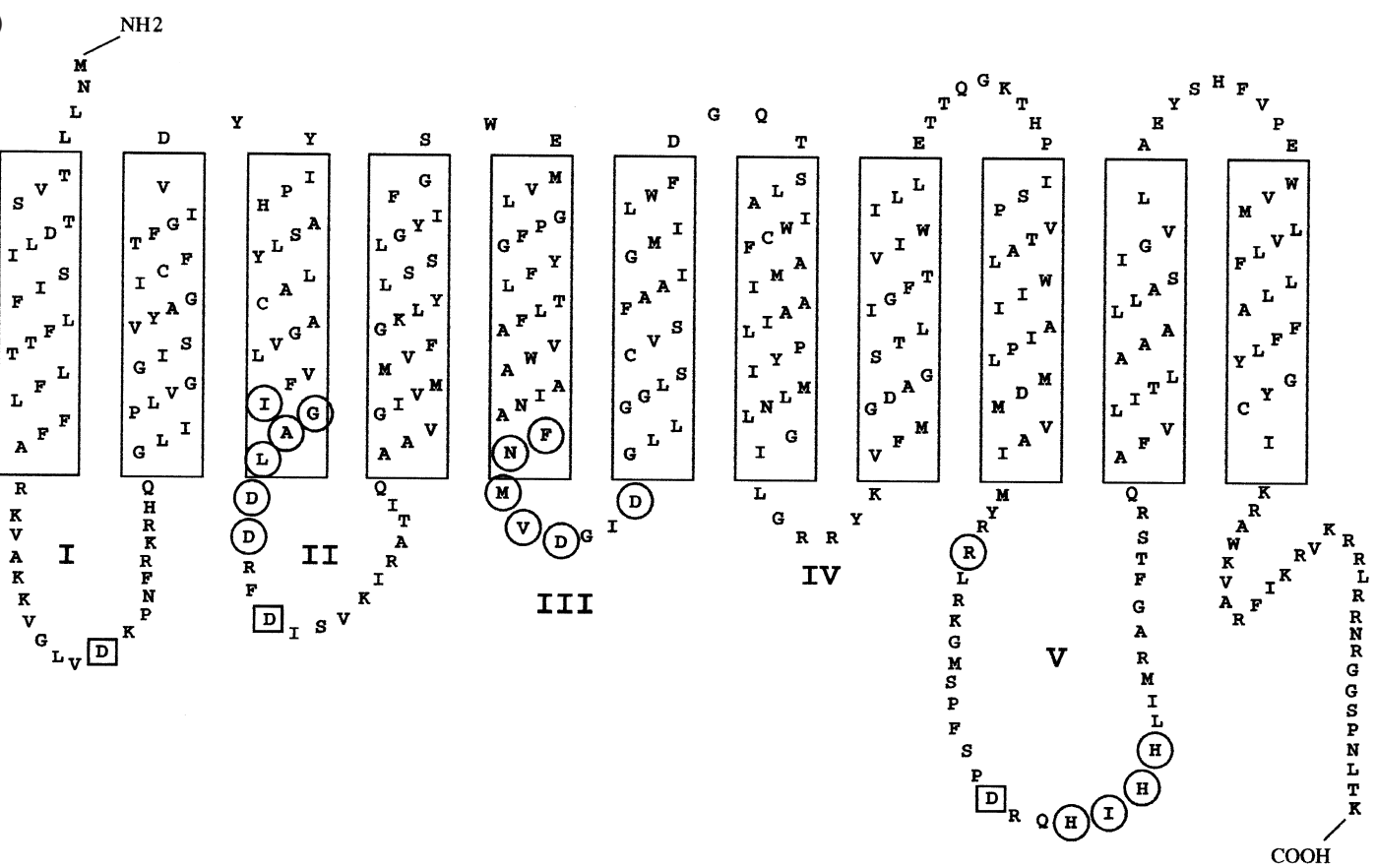

(b)
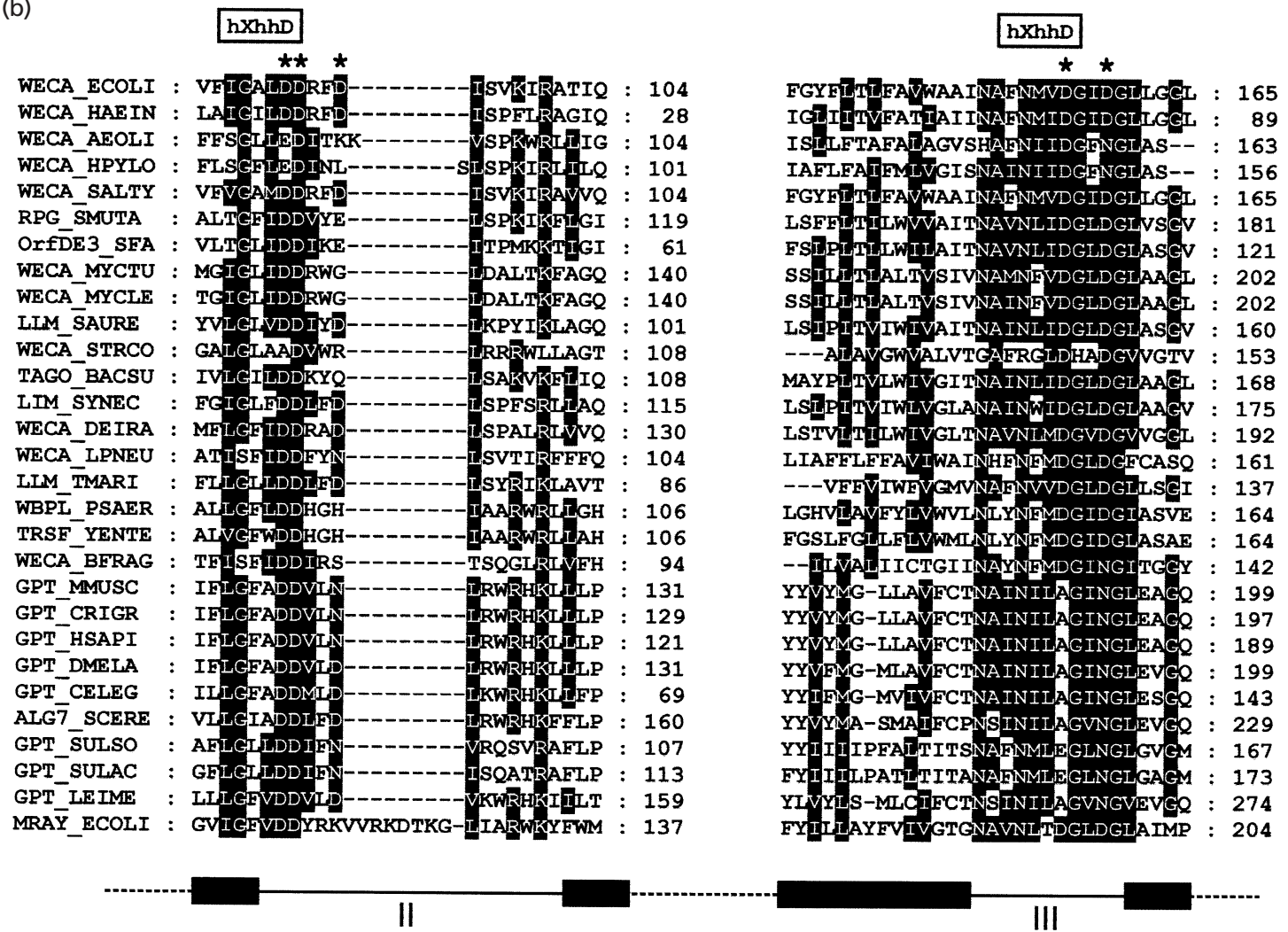

Fig. 1. (a) Topological model of E. coli WecA as predicted with TMHMM (Sonnhammer et al., 1998). The nontransmembrane segments that are exposed to the cytosol are indicated with roman numerals. Amino acids within circles denote those residues that are highly conserved within the WecA family [D90-D91 in cytosolic segment II, D156 and D159 in cytosolic segment III, R265 and HIHH279-282 in cytosolic segment V (Amer \& Valvano, 2001)]. Amino acids I86-G87A88-L89 and F152-N153-M154-V155, which together with D90 and D156, respectively, are characteristic of Walker B motifs, are also indicated. Additional aspartic acids in cytosolic segments, D35, D94 and D276, are indicated within 


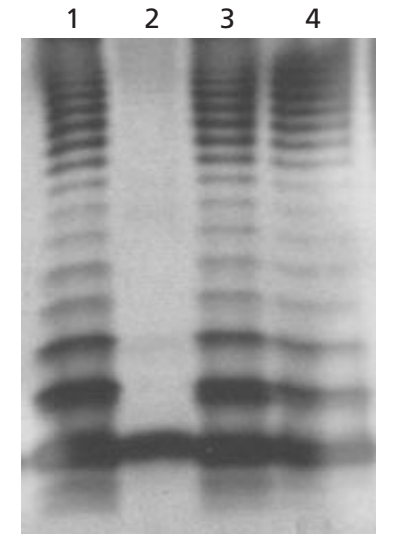

Fig. 2. Complementation of 07 LPS synthesis in strain MV501 by WecA proteins containing a modified C-terminal end. LPS samples from E. coli VW187 (wild-type O7 LPS) and MV501 (VW187 wecA::Tn10) were separated by SDS-PAGE followed by silver staining. Lanes: 1, VW187; 2, MV501 transformed with the vector control pGEM3; 3, MV501 transformed with pAA1 encoding the $E$. coli K-12 wild-type wecA; 4, MV501 transformed with pAA3, encoding wecA with a deletion that eliminates the last $27 \mathrm{C}$-terminal amino acids.

of in vitro transferase activity than the mutants DD156/159GG, DD156/159EE and D156G. Yet they did not complement the synthesis of O7 LPS in vivo, suggesting that regions II and III (Fig. 1), containing the conserved D90 and D91, as well as D156 and D159 are not functionally equivalent (see below).

The replacement of D94 did not affect the expression of O7 LPS (Fig. 4, lane 6) and membranes containing the mutated protein displayed an in vitro transfer activity that was comparable to that of the parental enzyme (Table 3). Mutation of D276 to glycine (D276G) also did not have any significant effect on WecA function, resulting in a protein that transfers GlcNAc (Table 3) and restores the formation of the characteristic O7 LPS ladder to levels comparable to $\mathrm{WecA}_{\mathrm{FLAG}}$ (Figs 4, lane 11). But mutant D35G led to a $53 \%$ reduction in the

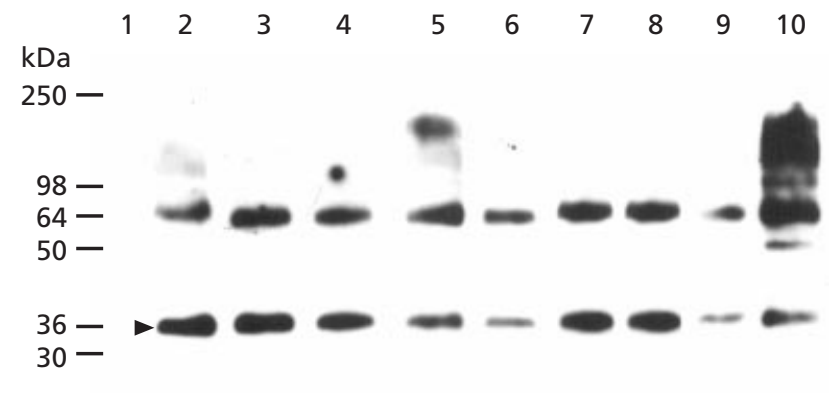

Fig. 3. Expression of the parental $W e c A_{F L A G}$ and mutant proteins. Bacterial cell membranes were prepared from strain MV501 expressing parental and mutated wecA genes, all tagged with the FLAG sequence. The Western blot was developed using mAb M2 anti-FLAG antibodies. Lanes: 1, pBAD24; 2, pAA26 (wecA $\left.A_{\mathrm{FLAG}}\right) ; 3$, pAA40 $\left(w e c A_{\mathrm{DD} 156 / 159 \mathrm{GG}}\right), 4$, pAA41 (wecA $\left.A_{\text {DD156/159EE }}\right) ; 5$, pAA43 $\left(w_{\text {ecA }} A_{\text {D156G }}\right) ; 6$, pAA45

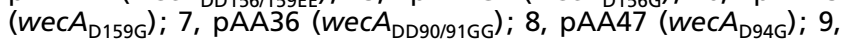
pAA35 (wecA $\left.A_{D 35 G}\right) ; 10$, pAA34 (wec $\left.A_{D 276 G}\right)$. The arrowhead indicates the WecA monomer. Polypeptides of higher molecular mass can be detected under the conditions utilized for protein denaturation, which represent oligomeric forms of WecA (Amer \& Valvano, 2000). The position of the following molecular mass standards is shown: myosin $(250 \mathrm{kDa})$, bovine serum albumin $(98 \mathrm{kDa})$, glutamic acid dehydrogenase $(64 \mathrm{kDa})$, alcohol dehydrogenase $(50 \mathrm{kDa})$, carbonic anhydrase $(36 \mathrm{kDa})$ and myoglobin (30 kDa).

transferase activity of WecA (Table 3), which correlated with a small but noticeable reduction in the level of LPS expression (Fig. 4, lane 3). $\mathrm{Wec}_{\mathrm{D} 35 \mathrm{G}}$ showed a lower level of expression in comparison to the other mutants and the parental $\mathrm{WecA}_{\mathrm{FLAG}}$ (Fig. 3, lane 9). Therefore, it is possible that this mutation affects the overall stability of the protein without a dramatic effect in its enzyme activity and it was not examined further.

The in vitro binding activity of mutant proteins was also investigated using a tunicamycin competition assay. Membranes containing $\mathrm{Wec}_{\mathrm{DD} 90 / 91 \mathrm{GG}}$ bound tunicamycin at levels comparable to $\mathrm{WecA}_{\mathrm{FLAG}}$ (Table 3 ). The lack of in vivo complementation and the low level of in

squares. (b) Alignment of prokaryotic and eukaryotic WecA homologues. WecA homologues were identified from a BLAST search using the $E$. coli WeCA protein as a query and aligned with the program CLUSTAL W. A partial alignment is shown of the region encompassing the conserved D90, D91, D94 residues (left) and the region containing the highly conserved D156 and D159 (right), indicated with asterisks. Boxes indicate the h-X-h-h-D motifs (see Discussion). The boundaries of transmembrane domains (filled boxes) and cytosolic loops II and III from the E. coli MraY protein are also indicated (Bouhss et al., 1999) at the bottom. Dotted lines denote the continuation of the amino acid sequence of the MraY protein, not shown in the figure. The sequences from the following organisms were compared (accession numbers in parentheses): WECA_ECOLI, E. coli (P24235); WECA_HAEIN, Haemophilus influenzae (P45341); WECA_AEOLI, Aquifex aeolicus (H70346); WECA_HPYLO, Helicobacter pylori strain 199 (B71801); WECA_SALTY, Salmonella typhimurium strain LT2 (AAF33469.1); RPG_SMUTA, Streptococcus mutans (BAA82114.1); OrfDE3_SFA, Streptococcus faecalis (AAC35916.1); WECA_MYCTU, Mycobacterium tuberculosis (Q10606); WECA_MYCLE, Mycobacterium leprae (P45830); LLM_SAURE, Staphylococcus aureus (A55856); WECA_STRCO, Streptomyces coelicolor (T36607); TAGO_BACSU, Bacillus subtilis (B69721); LIM_SYNEC, Synechocystis sp. (S74927); WECA_DEIRA, Deinococcus radiodurans (B75380); WECA_LPNEU, Legionella pneumophila (CAB65202.1); LLM_TMARI, Thermotoga maritima (H72238); WBPL_PSAER, Pseudomonas aeruginosa serotype O5 (AAC43404.1); TRSF_YENTE, Yersinia enterocolitica (S51265); WECA_BFRAG, Bacteroides fragilis (AAD40724.1); GPT_MMUSC, Mus musculus (NP_031901.1); GPT_CRIGR, Cricetulus griseus (P24140); GPT_HSAPI, Homo sapiens (NP_001373.1); GPT_DMELA, Drosophila melanogaster (AAF53252.1); GPT_CELEG, Caenorhabditis elegans (CAB60399.1); ALG7_SCERE, Saccharomyces cerevisiae (NP_009802.1); GPT_SULSO, Sulfolobus solfataricus (S75428); GPT_SULAC, Sulfolobus acidocaldarius (P39465); GPT_LEIME, Leishmania mexicana (P42864); MRAY_ECOLI, E. coli (P15876). 


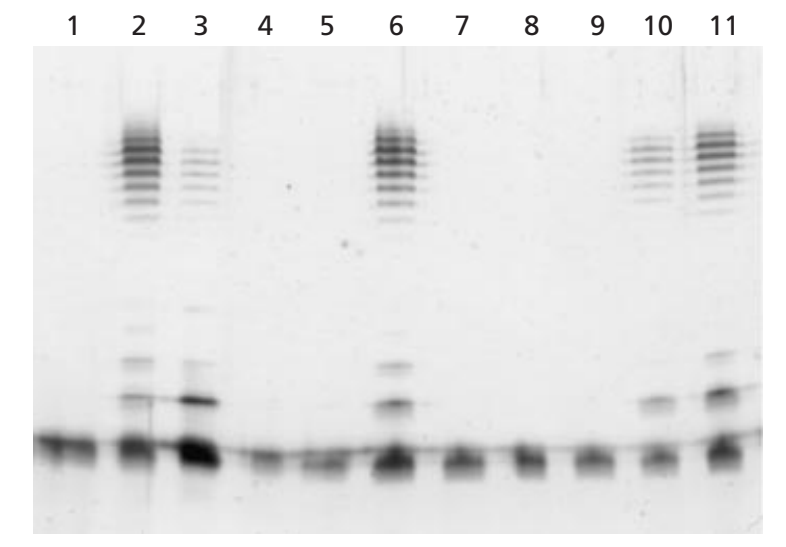

Fig. 4. $O 7$ LPS synthesis in strain MV501 containing the parental wec $A_{\text {FLAG }}$ and mutated wecA genes. LPS samples were prepared from $E$. coli MV501 cells transformed with plasmids encoding the parental WecA $A_{F L A G}$ and the mutated WecA forms. LPS was separated by SDS-PAGE followed by silver staining. Lanes: 1, pBAD24; 2 , pAA26 $\left(w_{\text {ec }} A_{\text {FLAG }}\right) ; 3$, pAA35 $\left(w e c A_{\text {D35G }}\right) ; 4$, pAA36 $\left(w e c A_{\mathrm{DD} 90 / 91 \mathrm{GG}}\right) ; 5, \mathrm{pAA} 37\left(w{ } A_{\mathrm{DD} 90 / 91 \mathrm{EE}}\right) ; 6, \mathrm{pAA} 47\left(w e c A_{\mathrm{D} 94 \mathrm{G}}\right)$; 7, pAA40 (wecA $\left.A_{\mathrm{DD} 156 / 159 \mathrm{GG}}\right) ; 8, \mathrm{pAA} 41\left(\right.$ wecA $\left._{\mathrm{DD} 156 / 159 \mathrm{EE}}\right) ; 9, \mathrm{pAA} 43$

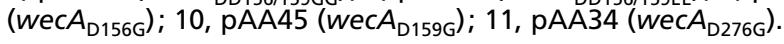

vitro transferase activity of $\mathrm{WecA}_{\mathrm{DD} 90 / 91 \mathrm{GG}}$ (Table 3 ), together with a normal substrate-binding activity in vitro suggests that these residues may be involved in interactions with the Und-P-P-GlcNAc reaction product. In contrast, WecA $\mathrm{DD}_{\mathrm{D} 156 / 159 \mathrm{GG}}$ and $\mathrm{WecA}_{\mathrm{D} 156 \mathrm{G}} \mathrm{did}$ not bind tunicamycin, while $\mathrm{WecA}_{\mathrm{D} 159 \mathrm{G}}$ showed reduced binding activity (Table 3 ). Since tunicamycin resembles the structure of the transition compound including UDPGlcNAc and the polyisoprenyl phosphate (Elbein, 1987), it was hypothesized that these amino acids could be involved in interactions with the substrates Und-P and UDP-GlcNAc. Alternatively, the loss of negative charges caused by the replacement of aspartic acids with glycine could potentially affect the local conformation of the catalytic site of the enzyme, and this change could be reflected in altered binding and transfer activities. But the conservative substitution of these aspartic acid residues with glutamic acid (DD90/91EE, DD156/ 159EE and D159E mutants) did not correct the defect (Table 3), suggesting that these aspartic acid residues, especially D156, may indeed be required for catalytic activity.

We utilized an independent assay to verify these results. During wzy-dependent polysaccharide chain biosynthesis, the transfer of the monosaccharide components of the $\mathrm{O}$ repeat occurs on the cytosolic side of the plasma membrane. This step is followed by the translocation or 'flipping' of the Und-P-P-saccharide from the cytoplasmic to the periplasmic surface of the plasma membrane, where the saccharide portion is ligated to preformed lipid A-core oligosaccharide. In a previous study, we have shown that translocation of GlcNAc-P-P-Und and ligation of this sugar to the lipid A-core oligosaccharide can be detected by an immunoblot assay using a digoxigenin-labelled wheat germ agglutinin, and that both WecA and Wzx are required for this process (Feldman et al., 1999). Thus, detection

Table 3. In vitro enzyme activity of $\mathrm{WeCA}_{\mathrm{FLAG}}$ and its mutated derivatives

\begin{tabular}{|c|c|c|c|}
\hline Membrane extract" & Transferase activity $\dagger$ & $\begin{array}{c}\text { Percentage } \mathrm{Wec}_{\mathrm{FLAG}} \text { transferase } \\
\text { activity }\end{array}$ & $\begin{array}{c}\text { Percentage } \mathrm{Wec}_{\mathrm{FLAG}} \text {-binding } \\
\text { activity } \ddagger\end{array}$ \\
\hline No WecA & $6 \pm 2$ & $9 \pm 2$ & $9 \pm 1$ \\
\hline $\mathrm{WecA}_{\mathrm{FLAG}}$ & $71 \pm 10$ & 100 & 100 \\
\hline D35G & $33 \pm 1$ & $47 \pm 1$ & ND \\
\hline DD90/91GG & $21 \pm 2$ & $30 \pm 3$ & $103 \pm 5$ \\
\hline DD90/91EE & $22 \pm 6$ & $31 \pm 9$ & $100 \pm 4$ \\
\hline D94G & $99 \pm 9$ & $139 \pm 13$ & ND \\
\hline D156G & $6 \pm 0 \cdot 4$ & $8 \pm 1$ & $12 \pm 1$ \\
\hline D156E & $7 \pm 1$ & $10 \pm 1$ & $\mathrm{ND}$ \\
\hline DD156/159GG & $3 \pm 1$ & $4 \pm 1$ & $11 \pm 3$ \\
\hline DD156/159EE & $9 \pm 3$ & $13 \pm 4$ & ND \\
\hline D159G & $14 \pm 4$ & $19 \pm 6$ & $40 \pm 5$ \\
\hline D274G & $97 \pm 10$ & $136 \pm 9$ & ND \\
\hline
\end{tabular}

* Membrane extracts were prepared from E. coli MV510 cells transformed with the vector control pBAD (no WecA present) or the various plasmids encoding parental and mutated forms of $\mathrm{WecA}_{\mathrm{FLAG}}$.

† The transfer assay measured the incorporation of radioactive GlcNAc into a butanol-extractable fraction as described in Methods. The results were expressed as $10^{-3} \times \mathrm{pmol}$ incorporated GlcNAc $(\mathrm{mg} \text { membrane protein })^{-1}$. The standard transfer reaction was conducted with a final concentration of $1 \mathrm{mM} \mathrm{MgCl}$ and the counts were normalized as described in Methods. Data represent the mean \pm SD of three independent experiments.

‡ The binding assay measured the incorporation of radioactive GlcNAc into a butanol-extractable fraction in competition with residual tunicamycin as described in Methods. The results, expressed as a percentage of the parental WecA $\mathrm{FLAG}_{\text {activity at } 1 \mathrm{mM} \mathrm{MgCl}}$ concentration using normalized counts, represent the mean \pm SD of three independent experiments. ND, Not determined. 

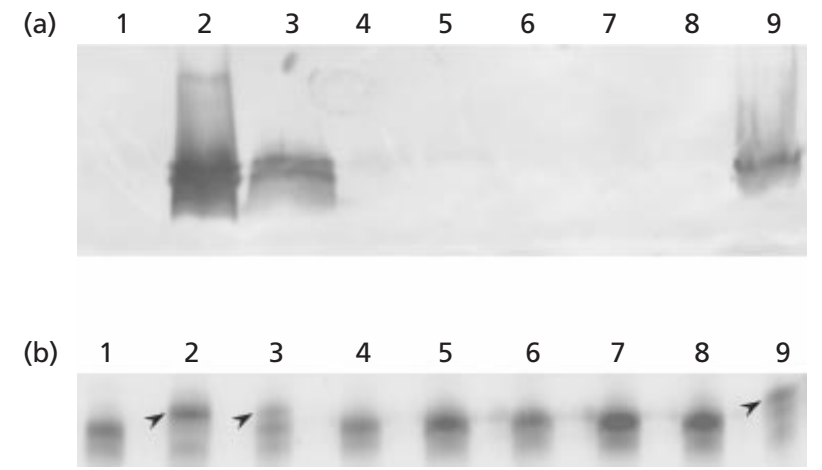

Fig. 5. Effect of aspartic acid substitutions on the translocation of Und-P-P-GICNAC and the ligation of GICNAc to lipid A-core oligosaccharide. (a) Immunoblot of LPS samples performed with DIG-labelled WGA and horseradish-peroxidase-labelled antidigoxigenin antibodies (Feldman et al., 1999). LPS samples were extracted from strain CLM20 containing pMF21 (encoding the wzx $\mathrm{O}$ antigen translocase) and the various plasmids encoding the parental and mutated WecA proteins. (b) Silver-stained SDS-Tricine PAGE using the same samples as in (a). Lanes: 1, pBAD24; 2, pAA26 $\left(w e c A_{\text {FLAG }}\right) ; 3$, pAA35 $\left(w e c A_{\text {D35G }}\right) ; 4$, pAA36 $\left(w^{2} A_{\mathrm{DD} 90 / 91 \mathrm{GG}}\right) ; 5, \quad$ pAA37 $\left.(w) A_{\mathrm{DD} 90 / 91 \mathrm{EE}}\right) ; 6, \quad$ PAA40 $\left(w e c A_{\mathrm{DD} 156 / 159 \mathrm{GG}}\right) ; 7, \quad \mathrm{pAA} 41 \quad\left(w e c A_{\mathrm{DD} 156 / 159 \mathrm{EE}}\right) ; 8, \quad \mathrm{pAA43}$ $\left(w e c A_{\mathrm{D} 156 \mathrm{G}}\right) ; 9$, pAA45 $\left(w e c A_{\mathrm{D} 159 \mathrm{G}}\right)$. Arrowheads indicate lipid Acore oligosaccharide complex of higher molecular mass due to the incorporation of a terminal GlcNAc (Feldman et al., 1999).

of a terminal GlcNAc with this assay represents the sum of activities involving the formation of Und-P-PGlcNAc, its translocation across the plasma membrane and the ligation of GlcNAc to lipid A-core oligosaccharide. We used the wheat-germ-agglutinin-binding assay to examine whether replacement of D90 and D91 interferes with this process. Fig. 5a, lanes 4 and 5 , shows that wheat germ agglutinin did not bind to the lipid A-core oligosaccharide from cells expressing either

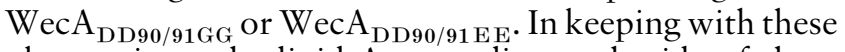
observations, the lipid A-core oligosaccharide of these cells has the same migration pattern as that of cells containing the control pBAD vector (Fig. 5b, lane 4). In contrast, cells expressing $\mathrm{WecA}_{\mathrm{FLAG}}$ displayed a positive reaction with wheat germ agglutinin and a slower migration of lipid A-core oligosaccharide in the gel (Figs $5 \mathrm{a}$ and $\mathrm{b}$, lanes 2 , respectively), denoting the addition of a terminal GlcNAc. Cells expressing $\mathrm{Wec}_{\mathrm{DD} 156 / 159 \mathrm{GG}}$, $\mathrm{Wec}_{\mathrm{DD} 156 / 159 \mathrm{EE}}$ and $\mathrm{WecA}_{\mathrm{D} 156 \mathrm{G}}$ did not have a terminal GlcNAc attached to lipid A-core oligosaccharide, in contrast to cells expressing $\mathrm{Wec}_{\mathrm{D} 159 \mathrm{G}}$ (Fig. 5a and b, lanes 6-9), supporting the data obtained from the in vitro transferase assay (Table 3).

\section{WecA enzyme activity requires metal ions}

The mechanism of phosphoryl transfer in several enzymes catalysing phosphoryl transfer reactions involves metal ions like $\mathrm{Mg}^{2+}$ or $\mathrm{Mn}^{2+}$ (Davies et al., 2000; Shemyakin et al., 1978) which interact with carboxylates within the protein (Allingham et al., 1999), such as aspartic acid or glutamic acid. The requirement

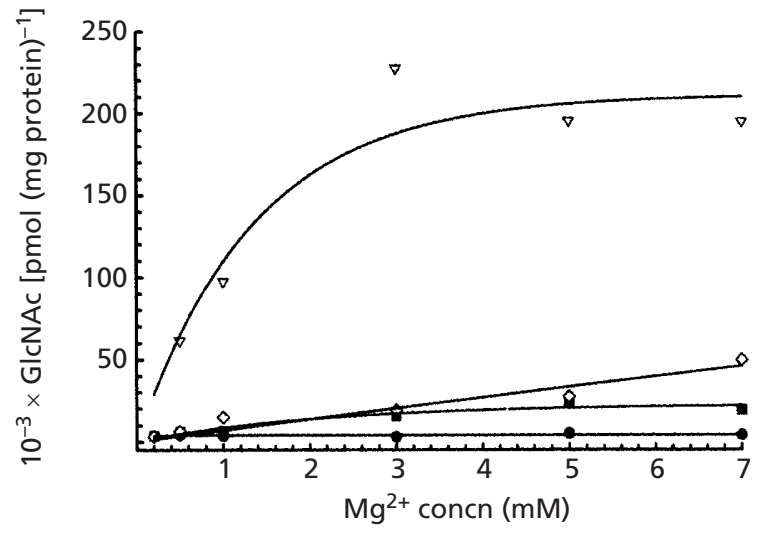

Fig. 6. Effect of magnesium ion concentration on in vitro GICNAC transferase activity. The GICNAC transferase activity of membranes obtained from cells containing $\operatorname{Wec}_{\mathrm{FLAG}}(\nabla)$, WecA $_{\text {DD 156/159EE }}(\boldsymbol{\square})$, WecA $_{\text {DD90/91EE }}(\diamond)$ and from cells transformed with the control plasmid pBAD24 (O) was measured at varying concentrations of $\mathrm{MgCl}_{2}$, as described in Methods. The results represent the mean of triplicate experiments. Standard deviations, ranging from 1 to $3 \%$ of the mean values, were too small to be represented by bars.

of metal ions for the enzymic activity of membranes containing $\mathrm{WecA}_{\mathrm{FLAG}}$ was determined by in vitro transfer assays in the presence of several cations at $1 \mathrm{mM}$ concentration. The results demonstrated that both $\mathrm{Mg}^{2+}$ and $\mathrm{Mn}^{2+}$ are necessary for enzymic activity, while there is no activity with $\mathrm{Ca}^{2+}, \mathrm{Zn}^{2+}$ or EDTA, a divalent ion chelator (data not shown). Furthermore, the enzymic activity of $\mathrm{WecA}_{\mathrm{FLAG}}$ membrane extracts increased proportionately with the $\mathrm{MgCl}_{2}$ concentration within a range from 0.5 to $3 \mathrm{mM}$, above which the activity was no longer enhanced (Fig. 6). From these results, we calculated that the parental enzyme required a concentration of $0.9 \mathrm{mM}$ for half-maximal transferase activity (Fig. 6). The DD90/91EE and DD156/159EE mutants also displayed a slow increase in enzymic activity following increasing $\mathrm{MgCl}_{2}$ concentrations (Fig. 6). However, the activities of membranes containing DD90/91EE and DD156/159EE mutants were too low to obtain an accurate fit of the data. Yet they approximately required at least 4 and $1.8 \mathrm{mM} \mathrm{MgCl}_{2}$ for halfmaximal activity, respectively (Fig. 6). The results suggest that the replacement of aspartic acid by glutamic acid residues in these proteins lowers their affinity for $\mathrm{Mg}^{2+}$ and is consistent with the possibility that these aspartic acids are involved in catalysis via interactions with the metal ion.

\section{DISCUSSION}

The present study reports the functional characterization of aspartic acid residues located within two regions of the WecA protein. The replacement of D90, D91 and D156 resulted in mutant forms of WecA that failed to function in vivo and exhibited a marked reduction in enzyme activity in vitro, suggesting that 
these residues may be involved in catalysis. The proposed function for these residues is in agreement with their predicted location in the plasma membrane/ cytosol interface, according to comparisons with the known topology of MraY, one of the members of the WecA family (Bouhss et al., 1999). Residues at this location could conceivably interact with soluble UDPGlcNAc as well as with the membrane-bound Und-P substrate. More importantly, these aspartic acids are conserved in the majority of the WecA homologues of prokaryotic and eukaryotic origin. Gross topological defects in the mutant proteins were not likely since control experiments for protein expression and membrane localization indicated that they were properly inserted in the plasma membrane and expressed at levels similar to the parental $\mathrm{WecA}_{\mathrm{FLAG}}$. Furthermore, the mutants carrying glutamic acid, a conservative substitution for aspartic acid, resulting in equivalent negative charges at the same positions, showed the same expression levels and low enzyme activities as the substitutions with glycine. But we cannot rule out at the present time that minor local topological defects around the mutated regions may occur. We have recently been able to solubilize the $\mathrm{WecA}_{\mathrm{FLAG}}$ protein, which also remains enzymically active. A more refined analysis of the parental WecA $\mathrm{FLAG}$ and its mutated derivatives awaits their solubilization and purification, currently under way in our laboratory.

The D90/91 and D156/159 mutants were not equivalent with respect to their binding activity as measured by a tunicamycin-binding competition assay. WecA $A_{\text {DD90/91GG }}$ displayed a binding activity similar to the parental WecA $_{\text {FLAG }}$, while WecA DD156/159GG $_{\text {phowed significantly }}$ decreased binding $\left(11 \%\right.$ of the parental WecA $\mathrm{WLAG}_{\mathrm{FL}}$ activity). A mutant WecA with a single substitution of the nearby D159 had an intermediate level of binding activity relative to that of $\mathrm{WecA}_{\mathrm{FLAG}}$, but this mutant protein could complement the synthesis of $\mathrm{O}$ antigen in vivo, despite a level of transferase activity in vitro reaching $19 \%$ of the $\mathrm{Wec}_{\mathrm{FLAG}}$ activity. In contrast, the transferase activity of DD90/91GG and DD90/91EE mutants was reduced to $30 \%$ of the parental WecA $\mathrm{WLAG}_{\mathrm{FLA}}$ activity. It has been proposed that tunicamycin acts as an inhibitor by resembling a compound similar to UDPGlcNAc and the isoprenoid phosphate (Elbein, 1987). Therefore, it is conceivable that the lack of binding activity in the DD156/159GG mutant identifies part of the enzyme's catalytic site. The functional characterization of mutants with substitutions in D90 and D91 suggest that this region may interact with the reaction product, Und-P-P-GlcNAc. This hypothesis is based on the fact that mutants in these residues had a higher level of transferase activity than the D156 and D159 mutants, but they displayed normal binding activity. We propose that this site may be involved in the release of Und-P-PGlcNAc, but more conclusive evidence using purified protein and reaction products is required to support this model. There is a precedent for a similar function in Salmonella enterica $\mathrm{WbaP}$ (formerly RfbP). This protein is a UDP-galactose:Und-P Gal-1-P transferase that mediates the initiation of $\mathrm{O}$ antigen biosynthesis in the major Salmonella serogroups (Wang et al., 1996). WbaP and WecA do not have any significant similarity in their primary amino acid sequence, nor in their predicted topology. But certain mutations causing amino acid substitutions in WbaP result in the lack of $\mathrm{O}$ antigen LPS on the bacterial cell surface while the galactosyltransferase in vitro activity is only slightly reduced (Wang et al., 1996). These investigators suggested that the mutated residues reveal a function involved in the release of the newly formed polyisoprenol-P-P-linked saccharide to the component or components involved in its membrane translocation (Wang et al., 1996). Our results showing that $\mathrm{WecA}_{\mathrm{DD} 90 / 91 \mathrm{GG}}$ cannot function in an in vivo translocation assay that measures the ligation of GlcNAc to the lipid A-core oligosaccharide in a Wzx (O antigen translocase)- and WecA-dependent fashion (Feldman et al., 1999), suggest a similar role for D90 and D91.

Structural information obtained from several different enzymes catalysing the formation of phosphodiester bonds suggests a mechanism of phosphoryl transfer that involves metal ions, such as $\mathrm{Mg}^{2+}$ or $\mathrm{Mn}^{2+}$ (Davies et al., 2000; Shemyakin et al., 1978), which interact with carboxylates within the protein (Allingham et al., 1999). We have shown in this study that a metal ion, such as $\mathrm{Mg}^{2+}$ or $\mathrm{Mn}^{2+}$, is required for WecA enzyme activity and we also demonstrated that the levels of enzyme activity vary as a function of the concentration of $\mathrm{Mg}^{2+}$. Conceivably, the aspartic acid residues identified as important for WecA catalytic activity, D90, D91 and D156, and to some extent D159, may also function in divalent metal ion binding to promote substrate binding and the formation of the phosphodiester bond between GlcNAc-1-P and Und-P. Our data, showing that the DD90/91EE and DD156/159EE mutants required an increased concentration of $\mathrm{Mg}^{2+}$ for reaching halfmaximal activity, support this conclusion since these findings are consistent with the idea that introduction of glutamic acid residues at these positions lowers the affinity of the protein for $\mathrm{Mg}^{2+}$. Similar behaviour has been reported for an aspartic mutant in the catalytic subunit of an E. coli ATP-dependent efflux pump (Zhou \& Rosen, 1999). Furthermore, the proposed model involving metal ion interactions with the aspartic acids is in part supported by the observation that D90 and D156 are components of two short domains, I-G-A-LD90 and F-N-M-V-D156, respectively, which both possesses the features ascribed to the Walker B-motif (van der Wolk et al., 1995). This motif is composed of a stretch of hydrophobic (h) amino acids with the sequence $h-X-h-h-D$ ( $X=$ any amino acid), from which the terminal aspartic acid was shown to be required for the coordination of the $\mathrm{Mg}^{2+}$ ion in enzymes possessing nucleotide-binding sites (van der Wolk et al., 1995). Both h-X-h-h-D motifs are highly conserved in the prokaryotic and eukaryotic WecA homologues examined in this study (Fig. 1).

In conclusion, our experiments have identified functionally important aspartic acid residues in two 
regions of the WecA protein that are presumably exposed to the cytosol. Our data demonstrate that both of these regions are important for catalytic activity and they may be part of the catalytic site of the enzyme. The formation of a phosphodiester bond between a sugar phosphate and a polyisoprenyl phosphate lipid carrier is a conserved reaction in prokaryotic and eukaryotic cells. Therefore, it is not surprising that the critical aspartic acid residues identified in this study, as well as the associated Walker B-motifs, are highly conserved in eukaryotic and prokaryotic homologues of this protein family, attesting to their essential functional roles. The purification of WecA, currently under way in our laboratory, will permit us to determine more precisely its enzymic properties, including the demonstration of direct metal-ion-binding, a necessary step to gain an increasing understanding of this enzyme.

\section{ACKNOWLEDGEMENTS}

This study was supported by grant MT-10206 from the Canadian Institutes of Health Research to M.A.V. A.O. A. was supported by an Ontario Graduate Scholarship Award. We thank Cristina Marolda, Tracey Hunt, David Haniford and Craig Strathdee for helpful discussions and advice, and Nancy Martin for the gift of anti-OmpA antiserum.

\section{REFERENCES}

Alexander, D. C. \& Valvano, M. A. (1994). Role of $r f e$ gene in the biosynthesis of the Escherichia coli O7-specific lipopolysaccharide and other $\mathrm{O}$-specific polysaccharides containing $\mathrm{N}$-acetylglucosamine. J Bacteriol 176, 7079-7084.

Allingham, J. S., Pribil, P. A. \& Haniford, D. B. (1999). All three residues of the $\mathrm{Tn} 10$ transposase DDE catalytic triad function in divalent metal ion binding. J Mol Biol 289, 1195-1206.

Altschul, S. F., Madden, T. L., Schaffer, A. A., Zhang, J., Zhang, Z., Miller, W. \& Lipman, D. J. (1997). Gapped BLAST and PSI-BLAST : a new generation of protein database search programs. Nucleic Acids Res 25, 3389-3402.

Amer, A. O. \& Valvano, M. A. (2000). The N-terminal region of the Escherichia coli WecA (Rfe) protein, containing three predicted transmembrane helices, is required for function but not for membrane insertion. J Bacteriol 182, 498-503.

Amer, A. O. \& Valvano, M. A. (2001). Conserved amino acid residues found in a predicted cytosolic domain of the lipopolysaccharide biosynthetic protein WecA are implicated in the recognition of UDP-N-acetylglucosamine. Microbiology 147, 3015-3025.

Anderson, M. S., Eveland, S. S. \& Price, N. P. (2000). Conserved cytoplasmic motifs that distinguish sub-groups of the polyprenol phosphate: $N$-acetylhexosamine-1-phosphate transferase family. FEMS Microbiol Lett 191, 169-175.

Barr, K., Nunes-Edwards, P. \& Rick, P. D. (1989). In vitro synthesis of a lipid-linked trisaccharide involved in synthesis of enterobacterial common antigen. J Bacteriol 171, 1326-1332.

Bouhss, A., Mengin-Lecreulx, D., Le Beller, D. \& Van Heijenoort, J. (1999). Topological analysis of the MraY protein catalysing the first membrane step of peptidoglycan synthesis. Mol Microbiol 34, 576-585.

Bronner, D., Clarke, B. R. \& Whitfield, C. (1994). Identification of an ATP-binding cassette transport system required for trans- location of lipopolysaccharide $\mathrm{O}$-antigen side-chains across the cytoplasmic membrane of Klebsiella pneumoniae serotype O1. Mol Microbiol 14, 505-519.

Burda, P. \& Aebi, M. (1999). The dolichol pathway of N-linked glycosylation. Biochim Biophys Acta 1426, 239-257.

Clarke, B. R., Bronner, D., Keenleyside, W. J., Severn, W. B., Richard, J. C. \& Whitfield, C. (1995). Role of Rfe and RfbF in the initiation of biosynthesis of D-galactan I, the lipopolysaccharide $\mathrm{O}$ antigen from Klebsiella pneumoniae serotype O1. J Bacteriol 177, 5411-5418.

Cserzo, M., Wallin, E., Simon, I., von Heijne, G. \& Elofsson, A. (1997). Prediction of transmembrane $\alpha$-helices in prokaryotic membrane proteins: the dense alignment surface method. Protein Eng 10, 673-676.

Dal Nogare, A. R. \& Lehrman, M. A. (1988). Conserved sequences in enzymes of the UDP-GlcNAc/MurNAc family are essential in hamster UDP-GlcNAc dolichol-P GlcNAc-1-P transferase. Glycobiology 8, 625-632.

Dan, N. \& Lehrman, M. A. (1997). Oligomerization of hamster UDP-GlcNAc: dolichol-P GlcNAc-1-P transferase, an enzyme with multiple transmembrane spans. J Biol Chem 272, 14214-14219.

Dan, N., Middleton, R. B. \& Lehrman, M. A. (1996). Hamster UDP$\mathrm{N}$-acetylglucosamine: dolichol-P $\mathrm{N}$-acetylglucosamine-1-P transferase has multiple transmembrane spans and a critical cytosolic loop. J Biol Chem 271, 30717-30724.

Datta, A. K. \& Lehrman, M. A. (1993). Both potential dolichol recognition sequences of hamster GlcNAc-1-phosphate transferase are necessary for normal enzyme function. J Biol Chem 268, 12663-12668.

Davies, D. R., Goryshin, I. Y., Reznikoff, W. S. \& Rayment, I. (2000). Three-dimensional structure of the Tn5 synaptic complex transposition intermediate. Science 289, 77-85.

Dower, W. J., Miller, J. F. \& Ragsdale, C. W. (1988). High efficiency transformation of E. coli by high voltage electroporation. Nucleic Acids Res 16, 6127-6145.

Elbein, A. D. (1987). Inhibitors of the biosynthesis and processing of N-linked oligosaccharide chains. Annu Rev Biochem 56, 497-534.

Feldman, M. F., Marolda, C. L., Monteiro, M. A., Perry, M. B., Parodi, A. J. \& Valvano, M. A. (1999). The activity of a putative polyisoprenol-linked sugar translocase (Wzx) involved in Escherichia coli $\mathrm{O}$ antigen assembly is independent of the chemical structure of the O repeat. J Biol Chem 274, 35129-35138.

Gaspar, J. A., Thomas, J. A., Marolda, C. L. \& Valvano, M. A. (2000). Surface expression of O-specific lipopolysaccharide in Escherichia coli requires the function of the TolA protein. Mol Microbiol 38, 262-275.

Guzman, L. M., Belin, D., Carson, M. J. \& Beckwith, J. (1995). Tight regulation, modulation, and high-level expression by vectors containing the arabinose PBAD promoter. J Bacteriol 177, 4121-4130.

Hanahan, D. (1983). Studies on transformation of Escherichia coli with plasmids. J Mol Biol 166, 557-580.

Klena, J. D. \& Schnaitman, C. A. (1993). Function of the $r f b$ gene cluster and the $r f e$ gene in the synthesis of $\mathrm{O}$ antigen by Shigella dysenteriae 1. Mol Microbiol 9, 393-402.

Lehrman, M. A. (1994). A family of UDP-GlcNAc/ MurNAc: polyisoprenol-P GlcNAc/MurNAc-1-P transferases. Glycobiology 4, 768-771.

Marolda, C. L., Welsh, J., Dafoe, L. \& Valvano, M. A. (1990). Genetic analysis of the O7-polysaccharide biosynthesis region 
from the Escherichia coli O7-K1 strain VW187. J Bacteriol 172, 3590-3599.

Meier-Dieter, U., Barr, K., Starman, R., Hatch, L. \& Rick, P. D. (1992). Nucleotide sequence of the Escherichia coli rfe gene involved in the synthesis of enterobacterial common antigen. $J$ Biol Chem 267, 746-753.

Osborn, M. J., Gander, J. E., Parisi, E. \& Carson, J. (1972). Mechanism of assembly of the outer membrane of Salmonella typhimurium. Isolation and characterization of cytoplasmic and outer membrane. J Biol Chem 247, 3962-3972.

Rick, P. D. \& Silver, R. P. (1996). Enterobacterial common antigen and capsular polysaccharides. In Escherichia coli and Salmonella: Cellular and Molecular Biology, pp. 104-122. Edited by F. C. Neidhardt, R. Curtiss III, J. L. Ingraham, E. C. C. Lin, K. B. Low, B. Magasanik, W. S. Reznikoff, M. Riley, M. Schaechter \& H. E. Umbarger. Washington, DC: American Society for Microbiology.

Rush, J. S., Rick, P. D. \& Waechter, C. J. (1997). Polyisoprenyl phosphate specificity of UDP-GlcNAc: undecaprenyl phosphate $\mathrm{N}$-acetylglucosaminyl 1-P transferase from E. coli. Glycobiology 7, 315-322.

Shemyakin, M. F., Malygin, A. G. \& Patrushev, L. I. (1978). A study of the conditions of the first phosphodiester bond formation by E. coli RNA polymerase. FEBS Lett 91, 253-256.

Sonnhammer, E. L. L., von Heijne, G. \& Krogh, A. (1998). A hidden Markov model for predicting transmembrane helices in protein sequences. In Proceedings of Sixth International Conference on Intelligent Systems for Molecular Biology, pp. 175-182. Edited by J. Glasgow, T. Littlejohn, F. Major, R. Lathrop, D. Sankoff \& C. Sensen. Menlo Park, CA: American Association for Artificial Intelligence Press.

Thompson, J. D., Higgins, D. G. \& Gibson, T. J. (1994). CLUSTAL W : improving the sensitivity of progressive multiple sequence align- ment through sequence weighting, position specific gap penalties and weight matrix choice. Nucleic Acids Res 22, 4673-4680.

Valvano, M. A. \& Crosa, J. H. (1989). Molecular cloning and expression in Escherichia coli $\mathrm{K}-12$ of chromosomal genes determining the $\mathrm{O} 7$ lipopolysaccharide antigen of a human invasive strain of E. coli O7: K1. Infect Immun 57, 937-943.

Wang, L., Lui, D. \& Reeves, P. R. (1996). C-terminal half of Salmonella enterica $\mathrm{WbaP}$ ( $\mathrm{RfbP}$ ) is the galactosyl-1-phosphate transferase domain catalysing the first step of $\mathrm{O}$ antigen synthesis. J Bacteriol 178, 2598-2604.

Whitfield, C. (1995). Biosynthesis of lipopolysaccharide O antigens. Trends Microbiol 3, 178-185.

van der Wolk, J. P. W., Klose, M., de Wit, J. G., den Blaauwen, T., Freudl, R. \& Driessen, A. J. (1995). Identification of the magnesium-binding domain of the high-affinity ATP-binding site of the Bacillus subtilis and Escherichia coli SecA protein. J Biol Chem 270, 18975-18982.

Yao, Z. \& Valvano, M. A. (1994). Genetic analysis of the O-specific lipopolysaccharide biosynthesis region $(r f b)$ of Escherichia coli K12 W3110: identification of genes that confer group 6 specificity to Shigella flexneri serotypes Y and 4a. J Bacteriol 176, 4133-4143.

Zhou, T. \& Rosen, B. P. (1999). Asp ${ }^{45}$ is a $\mathrm{Mg}^{2+}$ ligand in the ArsA ATPase. J Biol Chem 274, 13854-13858.

Zhu, X. \& Lehrman, M. A. (1990). Cloning, sequence, and expression of a cDNA encoding hamster UDP-GlcNAc: dolichol phosphate- $N$-acetylglucosamine-1-phosphate transferase. J Biol Chem 265, 14250-14255.

Received 3 August 2001; revised 1 October 2001; accepted 11 October 2001. 